\title{
First law of thermodynamics and Friedmann-like equations in braneworld cosmology
}

\author{
Xian-Hui Ge ${ }^{\text {a }}$ \\ ${ }^{a}$ Asia-Pacific Center for Theoretical Physics, \\ Pohang 790-784, Republic of Korea
}

\begin{abstract}
We derive the Friedmann-like equations in braneworld cosmology by imposing the first law of thermodynamics and Bekenstein's area-entropy formula on the apparent horizon of a Friedmann-Robertson-Walker universe in both Randall-Sundrum II gravity and Dvali-Gabadadze-Porrati gravity models. Israel's boundary condition plays an important role in our calculations in both cases, besides the first law of thermodynamics and Bekenstein's area-entropy formula. The results indicate that thermodynamics on the brane world knows the behaviors of gravity.
\end{abstract}

PACS:98.80.-k, 04.50.+h

\section{Introduction}

The relations between thermodynamics of space time and the nature of gravity is one of the most intriguing topics in theoretical physics. The four laws of black hole thermodynamics were first derived from the classical Einstein equation [1. With the discovery of Hawking radiation of black holes [2], it became clear that the analogy is actually an identity[3]. On the other hand, the Einstein equation is derived by Jacobson from the proportionality of entropy and horizon area together with the first law of thermodynamics $\delta Q=T d S[4$. Verlinde found that the Friedmann equation in a radiation dominated Friedmann-Robertson-Walker (FRW) universe can be written in an analogous form of the Cardy-Verlinde formula, an entropy formula for a conformal field theory [5]. The above observations imply that thermodynamics of space time and the Einstein equation are closely related [6]. In ref.[7], Cai and Kim derived the Friedmann equations of FRW universe with any spatial curvature by applying the first law of thermodynamics to the apparent horizon and assuming the geometric entropy is given by a quarter of the apparent 
horizon area.

The main interest of this paper relys on the relations between the first law of thermodynamics and the Friedmann-like equation in braneworld cosmology. In the study of three-brane cosmological models, an unusual law of cosmological expansion on the brane has been reported (for incomplete references see [8,9,10,11,12]). According to this law, the energy density of matter on the brane quadratically enters the right-hand of the new Friedmann equation for the braneworld cosmology, in contrast with the standard cosmology, where the Friedmann equation depends linearly on the energy density of matter. In this paper, we shall derive the Friedmann-like equation in the braneworld cosmology from the first law of thermodynamics.

This paper is organized as follows. In section 2, we discuss the relations between thermodynamics and gravity in Randall-Sundrum II (RSII) model [13. In section 3, we derive the Friedmann-like equation in Dvali-GabadadzePorrati (DGP) model [14] by applying the first law of thermodynamics to the apparent horizon together with Israel's boundary condition. We present our conclusions in section 4.

\section{FRW universe in Randall-Sundrum II gravity}

We consider a D-brane located on the boundary of a $(n+1)$-dimensional Anti-de Sitter space time, as our visible universe with ordinary matter being trapped on this brane by string theory effects. We will derive the braneworld cosmological evolution equations from the first law of thermodynamics. At each point of on the brane, we define a space-time unit normal, $N_{A}=N_{A}(x)$, to the surface that satisfies $g^{A B} N_{A} N_{B}=1 . g^{A B}$ is the bulk metric and the indices $A, B$ run over all the bulk coordinates. The bulk metric induces a metric on the brane,

$$
g_{\mu \nu}=g_{A B}-N_{A B} \text {. }
$$

The Einstein equation on the 3-brane world has been derived in[15]. The Einstein equation on this $(n-1)$-brane is given by [16,17] (we use the notations in [16]),

$$
{ }^{(n)} G_{\mu \nu}=-\Lambda_{n} g_{\mu \nu}+8 \pi G_{n} T_{\mu \nu}+\kappa_{n+1}^{4} \Pi_{\mu \nu}-E_{\mu \nu}
$$

where

$$
\begin{aligned}
\Lambda_{n} & =\kappa_{n+1}^{2}\left[\frac{n-2}{n} \Lambda_{n+1}+\frac{(n-2)}{8(n-1)} \kappa_{n+1}^{2} \lambda^{2}\right] \\
G_{n} & =\frac{n-2}{32 \pi(n-1)} \lambda \kappa_{n+1}^{4}, \\
\Pi_{\mu \nu} & =-\frac{1}{4} T_{\mu \alpha} T_{\nu}^{\alpha}+\frac{1}{4(n-1)} T T_{\mu \nu}+\frac{1}{8} g_{\mu \nu} T_{\alpha \beta} T^{\alpha \beta}-\frac{1}{8(n-1)} T^{2} g_{\mu \nu},
\end{aligned}
$$


where $\kappa_{n+1}^{2}=8 \pi G_{n+1}, \Lambda_{n}$ is the brane cosmological constant, $\lambda$ is the brane tension, $G_{n}$ is the Newton's constant in n-dimensions, $T_{\mu \nu}$ is the energymomentum tensor of matter in the brane world, and $E_{\mu \nu}$ is the Weyl tensor, which is vanishing in pure Anti-de Sitter space time. We can see from (2) that the n-dimensional effective equations of motion, being given entirely in terms of quantities defined on the brane, is independent of the evolution of the bulk space time. Different from the standard Einstein's equation in general relativity, the energy-momentum tensor in the right-hand of (2) is modified due to the presence of extrinsic curvature when project $(n+1)$-dimensional bulk quantities to the brane. We will not solve (2) in the rest of our work, but just utilize the modified energy-momentum tensor in the right-hand of (2) together with the first law of thermodynamics to derive cosmological evolution equation on the brane.

In the following calculation, we assume the cosmological constant $\Lambda_{n}$ vanishes, since we are mainly concern with the dynamical aspects of the braneworld cosmology while $\Lambda_{n}$ is a constant and does not vary with time. The induced metric on the brane is the FRW metric with the form,

$$
d s_{n}^{2}=-d \tau^{2}+a^{2}(\tau)\left(\frac{d r^{2}}{1-k r^{2}}+r^{2} d \Omega_{n-2}^{2}\right)
$$

where $d \Omega_{n-2}^{2}$ is the metric on an $(n-2)$-dimensional Euclidean unit space of constant curvature, $k=1,0,-1$ which corresponds to the unit sphere, plane, and hyperboloid respectively. The metric (6) can be rewritten as [18]

$$
d s^{2}=h_{a b} d x^{a} d x^{b}+\tilde{r}^{2} d \Omega_{n-2}^{2},
$$

where $\tilde{r}=a(\tau) r$ and $x^{0}=\tau, x^{1}=r$. The 2-dimensional metric $h_{a b}=$ $\operatorname{diag}\left(-1, \frac{a^{2}(\tau)}{1-k r^{2}}\right)$. Since the apparent horizon satisfies the equation $h^{a b} \partial_{a} \tilde{r} \partial_{b} \tilde{r}=$ 0 , we obtain the radius of the apparent horizon,

$$
\tilde{r}_{A}=\frac{1}{\sqrt{H^{2}+\frac{k}{a^{2}}}}
$$

where $H=\frac{\dot{a}}{a}$ is the Hubble parameter. For a dynamical space time, the apparent horizon is regarded as the horizon satisfying the Bekenstein areaentropy formula[19]. A unified law of black hole dynamics and relativistic thermodynamics is derived in spherically symmetric general relativity, where the gradient of the active gravitational energy $E$ determined by the Einstein's equation is divided into energy-supply and work terms [19]. Assume the matter on the brane is given by a homogeneous perfect fluid of density $\rho$ and pressure $p$, so that

$$
T_{\mu \nu}=(\rho+p) U_{\mu} U_{\nu}+p g_{\mu \nu} .
$$

Substituting the tensor back to Eq.(2) and keep in mind that $\Lambda_{n}$ and $E_{\mu \nu}$ are vanishing, we find that, 


$$
\begin{aligned}
\tilde{T}_{\nu}{ }^{\mu} & =T_{\nu}{ }^{\mu}+\frac{\kappa_{n+1}^{4}}{\kappa_{n}^{2}} \Pi_{\nu}{ }^{\mu} \\
& =\operatorname{diag}\left(-\rho-\frac{n-2}{8(n-1)} \frac{\kappa_{n+1}^{4}}{\kappa_{n}^{2}} \rho^{2}, p+\frac{n-2}{8(n-1)} \frac{\kappa_{n+1}^{4}}{\kappa_{n}^{2}} \rho(\rho+2 p), \ldots\right),
\end{aligned}
$$

where $\tilde{T}_{\nu}^{\mu}$ can be regarded as the total effective energy-momentum tensor on the brane and $\kappa_{n}^{2}=8 \pi G_{n}$ denotes Newton's constant. Now we would like to project the total effective energy-momentum tensor $\tilde{T}_{\nu}^{\mu}$ to the normal direction of the $(n-1)$-space and have it denoted as $T^{a b}$. Then, one may define the work density by [18,19],

$$
W=-\frac{1}{2} T^{a b} h_{a b},
$$

and the energy-supply vector is given by,

$$
\Psi_{a} \equiv T_{a}^{b} \partial_{b} \tilde{r}+W \partial_{a} \tilde{r}
$$

As noted in ref. [19], the work density at the apparent horizon may be viewed as the work done by the change of the apparent horizon and the energy-supply at the horizon is total energy flow through the apparent horizon. Thus the total change of energy on the apparent horizon can be written as,

$$
\nabla E=A \Psi+W \nabla V
$$

where $A=(n-1) \Omega_{n-1} \tilde{r}^{n-2}$ and $V=\Omega_{n-1} \tilde{r}^{n-1}$ are the area and the volume of the $(n-1)$-brane with radius $\tilde{r}$, and $\Omega_{n-1}=\pi^{(n-1) / 2} / \Gamma((n-1) / 2+1)$ is the volume of an $(n-1)$-dimensional unit ball. Eq.(13) is interpreted as unified first law [19].

Let us turn to calculating the heat flow $\delta Q$ through the apparent horizon during an infinitesimal time interval $d t$, while keep the volume of the brane stable, namely $\nabla V=0$. In this sense,

$$
d E \equiv A \Psi
$$

The heat flow $\delta Q$ through the apparent horizon is just the amount of energy crossing it during the time interval $d t$. Thus, $\delta Q=-d E$ is the change of the energy inside the apparent horizon. From (10), (11) and (12), we obtain the expression of work density and energy-supply,

$$
\begin{aligned}
& W=-\frac{1}{2}\left(-\rho+p+\frac{n-2}{4(n-1)} \frac{\kappa_{n+1}^{4}}{\kappa_{n}^{2}} \rho p\right) \\
& \Psi_{t}=-\frac{1}{2}(\rho+p) \dot{a} r-\frac{n-2}{8(n-1)} \frac{\kappa_{n+1}^{4}}{\kappa_{n}^{2}} \rho(\rho+p) \dot{a} r \\
& \Psi_{r}=\frac{1}{2}(\rho+p) a+\frac{n-2}{8(n-1)} \frac{\kappa_{n+1}^{4}}{\kappa_{n}^{2}} \rho(\rho+p) a
\end{aligned}
$$


We assume the entropy of the apparent horizon is described by the Bekenstein area formula,

$$
S=\frac{A}{4 G_{n}}
$$

with the horizon temperature,

$$
T=\frac{1}{2 \pi \tilde{r}_{A}}
$$

Calculating the amount of energy crossing the apparent horizon and applying the first law, $-d E=A \Psi d t=T d S$, we find

$$
\kappa_{n}^{2}(\rho+p)\left(1+\frac{n-2}{8(n-1)} \frac{\kappa_{n+1}^{4}}{\kappa_{n}^{2}} \rho\right)=-(n-2)\left(\dot{H}-\frac{k}{a^{2}}\right) .
$$

Integrating (20) together with the energy conservation equation on the brane,

$$
\dot{\rho}+(n-1) H(\rho+p)=0
$$

we finally obtain the cosmological evolution equation in the Randall-Sundrum II universe

$$
H^{2}+\frac{k}{a^{2}}=\frac{2 \kappa_{n}^{2}}{(n-1)(n-2)} \rho+\frac{\kappa_{n+1}^{4}}{4(n-1)^{2}} \rho^{2} .
$$

The above equation is exactly the Friedmann-like equation that describes the cosmological evolution of Randall-Sundrum II universe, where the cosmological constant is set to be vanishing here.

One should note that in the above calculations, we have simply use the Bekenstein entropy-area formula. In ref. [16,20], the authors started from the Friedmann-like equation on the braneworld together with the first law of thermodynamics $-d E=T d S+W d V$ and then derived the entropy expressions for the apparent horizon. The results show that the apparent horizon is proportional to the horizon area but with some corrections and only in the large horizon radius limit, the entropy obeys the $1 / 4$ area law. Here, we would like to justify the assumption about the usage of Bekenstein formula. In the above calculation, we have assumed during the time interval $d t$, the volume of the brane does not change, namely $d V \propto d \tilde{r}_{A}=0$. In other words, we have only considered the isometric process, $-d E=T d S$. Thus, if one starts from the Friedmann-like equation (22) and consider the isometric thermodynamical process, one can find that the apparent horizon entropy obeys the $1 / 4$ area law. In this sense, let us examine the expression of the surface gravity at the apparent horizon, which is generally given by,

$$
\kappa=\frac{1}{2 \sqrt{-h}} \partial_{a}\left(\sqrt{-h} h^{a b} \partial_{b} \tilde{r}\right)=-\frac{1}{\tilde{r}_{A}}\left(1-\frac{\dot{\tilde{r}}_{A}}{2 H \tilde{r}_{A}}\right) .
$$

The second term in the parenthesis is vanishing while $d \tilde{r}_{A}=\frac{d \tilde{r}_{A}}{d t} d t=0$. As a consequence, the temperature is read as $T=\frac{1}{2 \pi \tilde{r}_{A}}$. Therefore, an isometric 
thermodynamical process on the apparent horizon encodes the cosmological evolution equation. However, as suggested in ref.[16]20], this does not mean that the Bekenstein area formula used in the calculations above really reveal the relation between horizon entropy and horizon geometry, since the presence of an extra dimension cause the gravity here to be non-Einstein type. More explicit entropy expressions of the apparent horizon of FRW universe in RSII model are given in ref.[16].

In summary, If the entropy and temperature of the brane can be described by the apparent horizon, an isometric thermodynamical process on the apparent horizon should ensure the Bekenstein area formula established and from the first law of thermodynamics $-d E=T d S$, we are able to obtain the Friedmann-like equation for an $(n+1)$-dimensional Randall-Sundrum II universe with arbitrary spatial curvature.

\section{FRW universe in Dvali-Gabadadze-Porrati gravity}

In this section, we explore the relations between the first law of thermodynamics and cosmology based on the Dvali-Gabadadze-Porrati gravity model[14]. In this model, a 3-brane is embedded in a spacetime with an infinitesize extra dimension. The usual gravitational laws is obtained by adding to the action of the brane an Einstein-Hilbert term computed with the intrinsic curvature on the brane. Particularly, one recovers a standard four-dimensional (4D) Newtonian potential for small distances, whereas gravity is in a 5D regime for large distances. The cosmology of this model in the case of a 5D bulk was studied by Binétruy et al $8,9,14$. It is shown there that if the cosmological model contains a scalar curvature term in the action for the brane, besides the brane and bulk cosmological constraints, the presence of the scalar curvature term in the brane action can lead to a late-time acceleration of the universe even in the absence of any material form of dark energy [21.

The cosmology in a 5-dimensional bulk is described by the metric,

$$
d s^{2}=-n^{2}(\tau, y) d \tau^{2}+a^{2}(\tau, y)\left(\frac{d r^{2}}{1-k r^{2}}+r^{2} d \Omega_{2}^{2}\right)+b^{2}(\tau, y) d y^{2}
$$

where $d \Omega^{2}$ is the metric of a 2-dimensional Euclidean unit space and $k=0, \pm 1$. Since we mainly focus our attention on the hypersurface defined by $y=0[8$, which the world volume of brane is identify with that of our universe, it is important to examine the behavior of metric in the bulk. The metric (24) can be rewritten as 18

$$
d s^{2}=h_{a b} d x^{a} d x^{b}+\tilde{r}^{2} d \Omega_{2}^{2}
$$


where $\tilde{r}=a(\tau, y) r$ and $x^{0}=\tau, x^{1}=r$. The 3-dimensional metric $h_{a b}=$ $\operatorname{diag}\left(-n^{2}(\tau, y), \frac{a^{2}(\tau, y)}{1-k r^{2}}, b^{2}(\tau, y)\right)$. The apparent horizon is defined by[19],

$$
h^{a b} \partial_{a} \tilde{r} \partial_{b} \tilde{r}=0
$$

which denotes the radius of the apparent horizon,

$$
\tilde{r}_{A}=\frac{1}{\sqrt{\frac{\dot{a}^{2}}{a^{2} n^{2}}+\frac{k}{a^{2}}-\frac{a^{\prime 2}}{a^{2} b^{2}}}}
$$

Since $a(\tau, y), b(\tau, y)$ and $n(\tau, y)$ are functions of the fifth dimension coordinate $y$, the above apparent horizon is different from that of standard cosmology. Hereafter we choose $n^{2}(y=0)=1$, which implies that the time on the brane corresponds to standard cosmic time, and assume the fifth dimension is static $\dot{b}=0$ and set $b=1$. The 5 -dimensional Einstein equations in the bulk is given by,

$$
\tilde{G}_{A B} \equiv \tilde{R}_{A B}-\frac{1}{2} \tilde{R} \tilde{g}_{A B}=\kappa_{5}^{2} \tilde{T}_{A B},
$$

where $\kappa_{5}^{2}=8 \pi G_{5}, \tilde{R}_{A B}$ is the five-dimensional Ricci tensor and $\tilde{T}_{A B}$ is the energy-momentum tensor of matter. The energy-momentum tensor can be further decomposed into two parts,

$$
\tilde{T}_{B}^{A}=-\Lambda_{5}+\left.T_{B}^{A}\right|_{\text {brane }}
$$

where $\Lambda_{5}$ is the 5 -dimensional bulk cosmological constant and the second term is the energy-momentum tensor of the brane at $y=0$, which is given by,

$$
\left.T_{B}^{A}\right|_{\text {brane }}=-\lambda \delta_{B}{ }^{\mu} \delta_{\nu}{ }^{A} g_{\nu}{ }^{\mu} \delta(y)+\delta(y) \operatorname{diag}(-\rho, \mathrm{p}, \mathrm{p}, \mathrm{p}, 0),
$$

where $\lambda$ corresponds to brane tension (or cosmological constant) and the last term is the matter content on the brane $(y=0)$, which is assumed in the form of a perfect fluid for homogenous and isotropic universe on the brane. We emphasize that the brane tension can in principle be tuned to be zero [14,22]. In view of the brane tension do not vary with cosmic time $t$ and the dynamical aspects of the braneworld cosmology are our object, hereafter we follow the method of ref.[7] and focus mainly on the matter content of the brane in the calculations. One will see lately that an integration constant can be regarded as the brane tension, in case it does not vanish.

Now we project the energy-momentum tensor of the matter content of the brane to the normal direction of $(\tau, r, y)$ that is denoted as $T_{a}{ }^{b}$. Note that we assume there are no flows of matter along the fifth dimension and $\left.T_{y y}\right|_{\text {brane }}=0$. The work density and energy-supply vector defined in (11) and (12) here read as, 


$$
\begin{array}{ll}
W & =\frac{1}{2} \delta(y)(\rho-p), \quad \Psi_{t}=-\frac{1}{2} \delta(y)(\rho+p) \dot{a} r \\
\Psi_{r}=\frac{1}{2} \delta(y)(\rho+p) a, & \Psi_{y}=\frac{1}{2} \delta(y)(\rho-p) a^{\prime} r .
\end{array}
$$

At the time interval $d t$, the amount of energy crossing the apparent horizon is then,

$$
-d E=-A \Psi=\delta(y) A(\rho+p) H \tilde{r}_{A} d t .
$$

Since our discussions about the brane are confined to the hypersurface $y=$ 0 , and the volume of the brane is exactly that of our observing universe, then considering an isometric thermodynamical process across the apparent horizon, we can assume that the entropy and temperature denoted by the apparent horizon can be written as,

$$
S=\frac{A}{4 G_{(4)}}, \quad T=\frac{1}{2 \pi \tilde{r}_{A}}
$$

where $A=4 \pi \tilde{r}_{A}^{2}$ is the apparent horizon area. Using the first law of thermodynamics, $-d E=T d S$, together with (32), we find that at $y=0$,

$$
\kappa_{4}^{2}(\rho+p)=-2\left(\dot{H}-\frac{k}{a^{2}}-\frac{a^{\prime} \dot{a}^{\prime}}{a \dot{a}}+\frac{a^{\prime 2}}{a^{2}}\right) .
$$

The energy conservation equation on the brane is given by,

$$
\dot{\rho}+3 H(\rho+p)=0 .
$$

From (34) and (35), we finally obtain,

$$
H^{2}+\frac{k}{a^{2}}-\frac{a^{2}}{a^{2}}=\frac{\kappa_{4}^{2}}{3} \rho+C
$$

where $C$ is the integration constant that denotes the brane cosmological constant, namely $C=\Lambda_{4}$. The brane cosmological constant is given by Eq.(3) with $n=4$,

$$
\Lambda_{4}=\frac{\kappa_{5}^{2}}{6}\left(\Lambda_{5}+\frac{\kappa_{5}^{2}}{6} \lambda^{2}\right)
$$

The value of $\frac{a^{2}}{a^{2}}$ can be determined by using the Israel junction condition [23, 15, 16],

$$
K_{\mu \nu}^{+}=K_{\mu \nu}^{-}=-\frac{\kappa_{5}^{2}}{2}\left(T_{\mu \nu}-\frac{1}{3} g_{\mu \nu} T\right),
$$

where $K_{\mu \nu}=g_{\mu}^{\alpha} g_{\nu}^{\beta} \nabla_{\alpha} n_{\beta}$ denotes the extrinsic curvature of the brane, $T_{\mu \nu}$ is the matter energy-momentum tensor of the brane, and the superscript "+" and "-" refer to the "+" side $\left(\lim _{\mathrm{y} \rightarrow+0}\right)$ and "-" side $\left(\lim _{\mathrm{y} \rightarrow-0}\right)$ of the brane, respectively. As a consequence, we have

$$
\frac{a^{2}}{a^{2}}=\frac{\kappa_{5}{ }^{4}}{36} \rho^{2}
$$


where the $Z_{2}$ symmetry $y \leftrightarrow-y$ has been used here. Therefore, the cosmological evolution equation on the brane has the final form,

$$
H^{2}+\frac{k}{a^{2}}=\frac{\kappa_{4}^{2}}{3} \rho+\frac{\kappa_{5}^{4}}{36} \rho^{2}+\Lambda_{4}
$$

Eq.(40) is in agreement with Eq.(22 when $n=4$ and a non-vanishing cosmological constant . By using Eq.(37) and the relation (44) (see also [24,25]),

$$
\kappa_{4}^{2}=\frac{\kappa_{5}^{4}}{6} \lambda
$$

Eq.(40) can be written as

$$
H^{2}+\frac{k}{a^{2}}=\frac{\kappa_{5}^{2}}{6} \Lambda_{5}+\frac{\kappa_{5}^{4}}{36}(\rho+\lambda)^{2},
$$

which is the exact results of [9] with vanishing Weyl tensor. If an intrinsic curvature term is taken into accounted, then Israel's junction condition is modified and the value of $\frac{a^{\prime}}{a}$ is replaced by [26],

$$
\frac{a^{\prime}}{a}=-\frac{\kappa_{5}^{2}}{6} \rho^{2}+\frac{\kappa_{5}^{2}}{2 \kappa_{4}^{2}}\left(H^{2}+\frac{k}{a^{2}}\right) .
$$

Substituting the above formula back to (36) and using (37) and (41), we obtain cosmological evolution equation in DGP gravity,

$$
H^{2}+\frac{k}{a^{2}}=\left(\sqrt{\frac{\kappa_{4}^{2}}{3} \rho_{b}+\frac{1}{4 r_{c}^{2}}}+\frac{1}{2 r_{c}}\right)^{2},
$$

where $\rho_{b}=\rho+\lambda, r_{c}=\frac{\kappa_{5}^{2}}{2 \kappa_{4}^{2}}$ and $\Lambda_{5}$ here has been set to be zero. Eq.(44) is precisely the Friedmann-like equation for a FRW universe in DGP gravity first found in ref.[26], when intrinsic curvature is added to the brane.

Therefore, to derive the Friedmann-like equations for FRW universe in DGP gravity, Israel's boundary condition is needed, besides applying the first law of thermodynamics and Bekenstein's entropy-area formula to the apparent horizon. The intrinsic curvature term added to the brane action plays a crucial role in obtaining (44).

\section{Conclusions}

In conclusion, we have presented an alternative methods to derive cosmological evolution equations in braneworld cosmology. Employing the first law of thermodynamics, $-d E=T d S$ and Bekenstein's area-entropy formula to 
the apparent horizon of FRW universe in RSII gravity and DGP gravity, we have obtained the Friedmann-like equations for brane world cosmology. Comparing section 2 with section 3, we find that Israel's boundary condition plays an important role in determining the effective energy-momentum tensor $\tilde{T}_{\mu \nu}$ or the apparent horizon $\tilde{r}_{A}$. Israel's boundary condition is not directly used in section 2 , since we were start with the brane world Einstein equation, but it is indeed crucial to derive the effective energy-momentum tensor $\tilde{T}_{\mu \nu}[15]$. An isometric thermodynamical process happened across the apparent horizon is assumed in the above discussions, thus whether a more general thermodynamical process yields the Friedmann-like equations or not worth further investigations. The detailed thermodynamical properties of apparent horizon in braneworld scenario are investigated in ref.[16,20] and some new physics about the braneworld gravity and thermodynamics is found.

It has been proved that gravity knows thermodynamics, when Bekenstein and Hawking found that entropy of black hole horizon is proportional to its horizon area [2,3]. Black hole entropy and temperature can be regarded as purely geometric quantities in that the horizon area and surface gravity is determined by space-time geometry. The above analysis and references mentioned [4,5,6,7,19], also indicate that thermodynamics knows gravity. Once the form of energy-momentum tensor of the system is determined, one can derive the spacetime geometric evolution equations from Eqs.(15) and (19). The results obtained are certainly related to the holographic principle, since the brane world cosmology models are closely related to the AdS/CFT conjecture [27]. It would be worthwhile to study the implications of above analysis to the holographic principle or quantum gravity in the future.

\section{Acknowledgements}

The author would like to thank Prof. S. P. Kim for his helpful discussions.

\section{References}

[1] J. M. Bardeen, B. Carter and S.W. Hawking, Comm. Math. Phys. 31 (1973) 161

[2] S. W. Hawking,Comm. Math. Phys. 43 (1975) 199

[3] J. D. Bekenstein, Phys. Rev. D 7 (1973) 2333

[4] T. Jacobson, Phys. Rev. Lett. 75 (1995) 1260 [gr-qc/9504004]

[5] E. Verlinde, hep-th/0008140 
[6] T. Padmanabhan, Class. Quantum Grav. , 19 (2002) 5387 gr-qc/0204019; T. Padmanabhan, Phys. Rep. 406 (2005) 49 gr-qc/0311036]; A. Paranjape, S. Sarkar, T. Padmanabhan, Phys.Rev., D 74,104015 (2006) hep-th/0607240]; A. Mukhopadhyay, T. Padmanabhan , Phys. Rev., D 74 (2006) 124023 [hep-th/0608120]; T. Padmanabhan and A. Paranjape, Phys. Rev. D75 (2007) 064004 gr-qc/0701003] ; R. G. Cai and L. M. Cao, Phys. Rev. D 75 (2007) 064008

[7] R. G. Cai and S. P. Kim, JHEP 02 (2005) 050

[8] P. Binétruy, C. Deffayet,and D. Langlois, Nucl. Phys. B565 (2000) 269 hep-th/9905012

[9] P. Binétruy, C. Deffayet, U. Ellwanger and D. Langlois, Phys. Lett. B 477 (2000) 285 hep-th/9910219

[10] H. Collins and B. Holdom, Phys. Rev. D62 (2000) 105009 hep-ph/0003173

[11] C. Barceló and M. Visser, Phys. Lett. B 482 (2000) 183 hep-th/0004056

[12] Y. V. Shtanov, hep-th/0005193

[13] L. Randall and R. Sundrum, Phys. Rev. Lett. 83 (1999) 4690

[14] G. Dvali, G. Gabadadze, M. Porrati, Phys. Lett. B 485 (2000) 208 hep-th/0005016

[15] T. Shiromiza, K. Maeda, and M. Sassaki, Phys. Rev. D 62 (2000) 349 |gr-qc/9910076]; A. N. Aliev and A. E. Gumrukcuoglu, Class. Quant. Grav. 21 (2004) 5081

[16] R. G. Cai and L. M. Cao, hep-th/0612144

[17] A. Padilla, Ph. D Thesis, hep-th/0210217.

[18] D. Bak and S. J. Rey, Class. Quantum Grav. 17 (2000) L83 hep-th/9902173

[19] S. A. Hayward, S. Mukohyama and M. C. Ashworth, Phys. Lett. A 256 (1999) 347 gr-qc/9810006; S. A. Hayward, Class. Quantum Grav. 15 (1998) 3147 gr-qc/9710089

[20] A. Sheykhi, B. Wang and R. G. Cai, hep-th/0701198; A. Sheykhi, B. Wang and R. G. Cai, hep-th/0701261

[21] C. Deffayet, G. Dvali and G. Gabadadze, Phys. Rev. D 65, ( 2002) 044023; C. Deffayet, S. J. Landau, J. Raux, M. Zaldarriaga and P. Astier, Phys. Rev. D 66 (2002) 024019 astro-ph/0201164

[22] G. Dvali and G. Gabadadze, Phys. Rev. D 63 (2001) 065007 hep-th/0008054

[23] W. Israel, Nuovo Cimento B 44 (1966) 1

[24] C. Csáki, M. Graesser and C. Kolda, J. Terning, Phys. Lett. B 462 (1999) 34 hep-th/9906513 
[25] J. M. Cline, C. Grosjean and G. Servant, Phys. Rev. Lett. 83 (1999) 4245 hep-ph/9906523

[26] C. Deffayet, Phys. Lett. B 502 (2001) 199

[27] J. M. Maldacena, Adv. Theor. Math. Phys. 2 (1998) 231 hep-th/9711200 